\title{
Spinal Subarachnoid Hemorrhage Induced Intractable Miotic Pupil. A Reminder of Ciliospinal Sympathetic Center Ischemia Based Miosis: An Experimental Study
}

\author{
Mehmet Dumlu AYDIN ${ }^{1}$, Ayhan KANAT ${ }^{2}$, Coskun YOLAS ${ }^{3}$, Celalettin SOYALP ${ }^{4}$, Mehmet Resid ONEN 5 , \\ Ilhan YILMAZ $^{6}$, Nuh Cagri KARAAVCI ${ }^{1}$, Muhammet CALIK ${ }^{7}$, Orhan BAYKAL ${ }^{8}$, Leyla RAMAZANOGLU ${ }^{9}$ \\ ${ }^{1}$ Ataturk University, Faculty of Medicine, Department of Neurosurgery, Erzurum, Turkey \\ ${ }^{2}$ Recep Tayyip Erdogan University, Faculty of Medicine, Department of Neurosurgery, Rize, Turkey \\ ${ }^{3}$ Erzurum Education and Research Hospital, Department of Neurosurgery, Erzurum, Turkey \\ ${ }^{4}$ Yuzuncu Yil University, Faculty of Medicine, Department of Anesthesiology, Van, Turkey \\ ${ }^{5}$ Umraniye Education and Research Hospital, Department of Neurosurgery, Istanbul, Turkey \\ ${ }^{6}$ Sisli Hamidiye Etfal Education and Research Hospital, Department of Neurosurgery, Istanbul, Turkey \\ ${ }^{7}$ Ataturk University, Faculty of Medicine, Department of Pathology, Erzurum, Turkey \\ ${ }^{8}$ Ataturk University, Faculty of Medicine, Department of Opthalmology, Erzurum, Turkey \\ ${ }^{9}$ Fatih Sultan Mehmet Education and Research Hospital, Department of Neurosurgery, Istanbul, Turkey
}

Corresponding author: Mehmet Dumlu AYDIN nmda11@hotmail.com

\section{ABSTRACT}

AIM: To examine ischemic neurodegeneration of the ciliospinal center on permanent miosis following subarachnoid hemorrhage $(\mathrm{SAH})$.

MATERIAL and METHODS: Nineteen rabbits were examined in this study. The animals were divided into three groups, as control $(G I, n=5)$, sham (GII, $n=5$ ) and study group (GIII, $n=9$ ). Pupil diameters were measured after giving 0.5 mL physiological saline for sham and autologous arterial blood for the study group into the cervico-thoracic subarachnoid space. After three weeks of follow up, the cervico-thoracic cord and bilateral superior cervical sympathetic ganglia were removed. The pupil diameter values were compared with degenerated neuron volumes of sympathetic ganglia and degenerated neuron densities of thoracic sympathetic nuclei which were studied by stereological methods.

RESULTS: The mean pupil diameter was $5180 \pm 370 \mu \mathrm{m}$ and the mean degenerated neuron density of the ciliospinal center was 4 $\pm 1 / \mathrm{mm}^{3}$ in animals of the control group (Gl). These values were $9850 \pm 610 \mu \mathrm{m}, 10 \pm 3 / \mathrm{mm}^{3}$ in sham (GII), and $7.010 \pm 440 \mu \mathrm{m}$ and $98 \pm 21 / \mathrm{mm}^{3}$ in the study (GIII) groups. There was an inverse relationship between degenerated neuron density of the ciliospinal nuclei and pupil diameters.

CONCLUSION: We showed and reported for the first time that ciliospinal sympathetic center ischemia-induced neurodegeneration may have been responsible for permanent miosis following $\mathrm{SAH}$.

KEYWORDS: Subarachnoid hemorrhage, Miotic pupil, Ciliospinal sympathetic center, Mydriasis, Miosis, Rabbit

Mehmet Dumlu AYDIN (1) : 0000-0002-0383-9739 Ayhan KANAT Coskun YOLAS

Celalettin SOYALP
(1): : 0000-0002-8189-2877

(D): :0000-0002-4888-4503

(1D) : 0000-0002-2687-5329
Mehmet Resid ONEN (D) : 0000-0003-4353-9068 Ilhan YILMAZ (1): 0000-0001-5548-2228 Nuh Cagri KARAAVCI (D): 0000-0002-4316-3614 Muhammet CALIK (D): 0000-0003-4553-6282
Orhan BAYKAL (D): 0000-0001-8321-1767 Leyla RAMAZANOGLU (D) : 0000-0001-9724-0198 


\section{INTRODUCTION}

A $n$ intracranial aneurysm occurs in $5 \%$ to $6 \%$ of the general population (10). Its rupture leads to subarachnoid hemorrhage $(\mathrm{SAH})$, which is a devastating condition (17). SAH accounts for approximately $5-10 \%$ of strokes (20), and currently, neurosurgery has gone through moments of great renewal (8). However, $\mathrm{SAH}$ has the highest mortality and morbidity among all types of stroke (23). Despite major improvements in surgical techniques for aneurysmal SAH, 30day mortality from SAH has been shown to have changed very little from what it was 40 years ago $(1,11)$.

Multiple ocular functions are controlled by the autonomic nervous system (21). Both parasympathetic and sympathetic nervous system disorders appear to be important factors in pupillary diameter changes (21). Although the basic mechanism of mydriasis is well known, the effects of denervative degeneration injury of cervical sympathetic ganglia induced by the spinal sympathetic center have not been investigated following SAH. One of the most common external physical signs of SAH is pupillary diameter abnormalities (21). Postganglionic fibers of the ciliary ganglia (CG) influence pupillodilatatory parasympathetic innervation, and postganglionic fibers coming from the superior cervical ganglion (SCG) command the sympathetic innervations (21). Postganglionic fibers of the SCG are under control of the ciliospinal center located in the cervicothoracic region. SCG neurons are responsible for accommodation and pupil constriction. However, pupil dilation is realized via SSG neurons. The sympathetic preganglionic pupil-dilation neurons are located at the C8-T1 segmental part of the spinal cord. While coming into the eye, these axons visit the choroid and innervate the dilator muscle of the iris (16). Neurodegeneration in the $\mathrm{CG}$, as a result of $\mathrm{SAH}$, induces indirect mydriasis (3). Vessels of cisternal segments of the oculomotor nerve are affected and parasympathetic preganglionic denervation of the CG may result in mydriasis due to plegia of the pupil-constrictor muscles (2). Highly degenerated neuron density of the CG and/or high neuron density of pupillodilatatory superior cervical sympathetic ganglia play an important role in pupil dilatation (19). SAH-induced ischemic degradation in the oculomotor nerve can result in indirect mydriasis because the presynaptic supply of CG via the preganglionic fibers fails to maintain its trophic effect (4). Although Onen et al. declared that spinal cord ischemia can be responsible for sympathetic center ischemia-related miosis (19), the main neuropathological mechanism of ciliospinal center ischemiabased miosis is not yet known. Nevertheless, Kanat et al. showed that spinal SAH results in severe spinal cord ischemia causing neurodegeneration in the dorsal root ganglion (DRG) via vasospasm of DRG-supplying arteries (12). Cerebral vasospasm is one of the most challenging complications following SAH (22). Vasospastic blood vessels of the spinal cord come across to subarachnoid blood and the vasospasm of DRG blood vessels may cause ischemic neurodegeneration in the DRG, while diminished normal neuron density (NND) or increased degraded neuron density (ND) of DRGs may play an essential role on the modulation of spinal cord blood flow. Low ND of DRG should be considered an essential factor in the pathogenesis of severe neurodegeneration in ciliospinal center ischemia in SAH. A previous experimental study exhibited that degenerated ND of the DRG play a role in vasospasm of the anterior spinal artery in $\mathrm{SAH}$. The objective of this study was to evaluate whether there was an association between ciliospinal center injury and miosis development after SAH.

\section{MATERIAL and METHODS}

Nineteen rabbits were used in this study. They were randomly separated into 3 groups of control (GI, $n=5)$, SHAM (GIl, $n=5$ ) and the study group (GIII, $n=9$ ). Experiments were done according to the procedures set by the ethics committee of Ataturk University Faculty of Medicine (B.30 .2.ATA.0.23.85-41/28.6.2010). The pupil diameters of all the animals were measured in sunlight using ocular tomography on the first day of the experiment (Figure 1). These results were accepted as the baseline control values. Pupil diameters were measured again after the injection of $0.5 \mathrm{~mL}$ saline to the sham group and autologous arterial blood into the cervicothoracic subarachnoid space of the study group. After a 3-week followup period, the cervicothoracic cord was extracted. The pupil diameter values were compared with the degenerated neuron densities of the ciliospinal center which were examined using stereological methods.

All animals were followed up for 3 weeks with daily pupillary diameter measurements. Thoracic sympathetic nuclei were examined histopathologically as described by Onen et al. (19). The physical dissector method was applied to estimate the number of degraded neurons in ciliospinal sympathetic nuclei as was described by Aydin et al (2).

\section{Statistical Analysis}

The data were analyzed using the Kruskal-Wallis and nonparametric Mann-Whitney $U$ tests. Differences were considered statistically significant at $p<0.005$.

\section{RESULTS}

The pupil diameter was $5180 \pm 370 \mu \mathrm{m}$, and the mean degraded neuron volume of the thoracic sympathetic center was $2 \pm 1 / \mathrm{mm}^{3}$ in animals of the control groups (Figure 1). These values were $9850 \pm 610 \mu \mathrm{m}$, and $9 \pm 3 / \mathrm{mm}^{3}$ in the sham group (Figure 2) and $7010 \pm 440 \mu \mathrm{m}$ and $98 \pm 77 / \mathrm{mm}^{3}$ in the study group (Figure 3). The macroscopic appearance of the brains and spinal cords of the study group showed subarachnoid hemorrhage-induced brain and spinal cord edema, and clot formation in the subarachnoid spaces and nerve roots. The basal view of a subarachnoid hemorrhage in a rabbit brain and extension of the spinal cord (base) (Figure $4 \mathrm{~A}, \mathrm{~B})$, and a horizontal section of the cervicothoracic spinal cord and sympathetic ciliospinal center neurons are shown in Figure $4 \mathrm{~A}, \mathrm{~B}$, respectively.

Apoptotic neuronal degradation was detected using the TUNEL method in ciliary ganglia (34\%), but apoptotic degeneration was not significant in the thoracic sympathetic center as it is supplied by external spinal arteries which are rarely affected by $\mathrm{SAH}$-related vasospasm (Figure $5 \mathrm{~A}, \mathrm{~B}$ ). 


\section{OCULUS - PENTACAM}

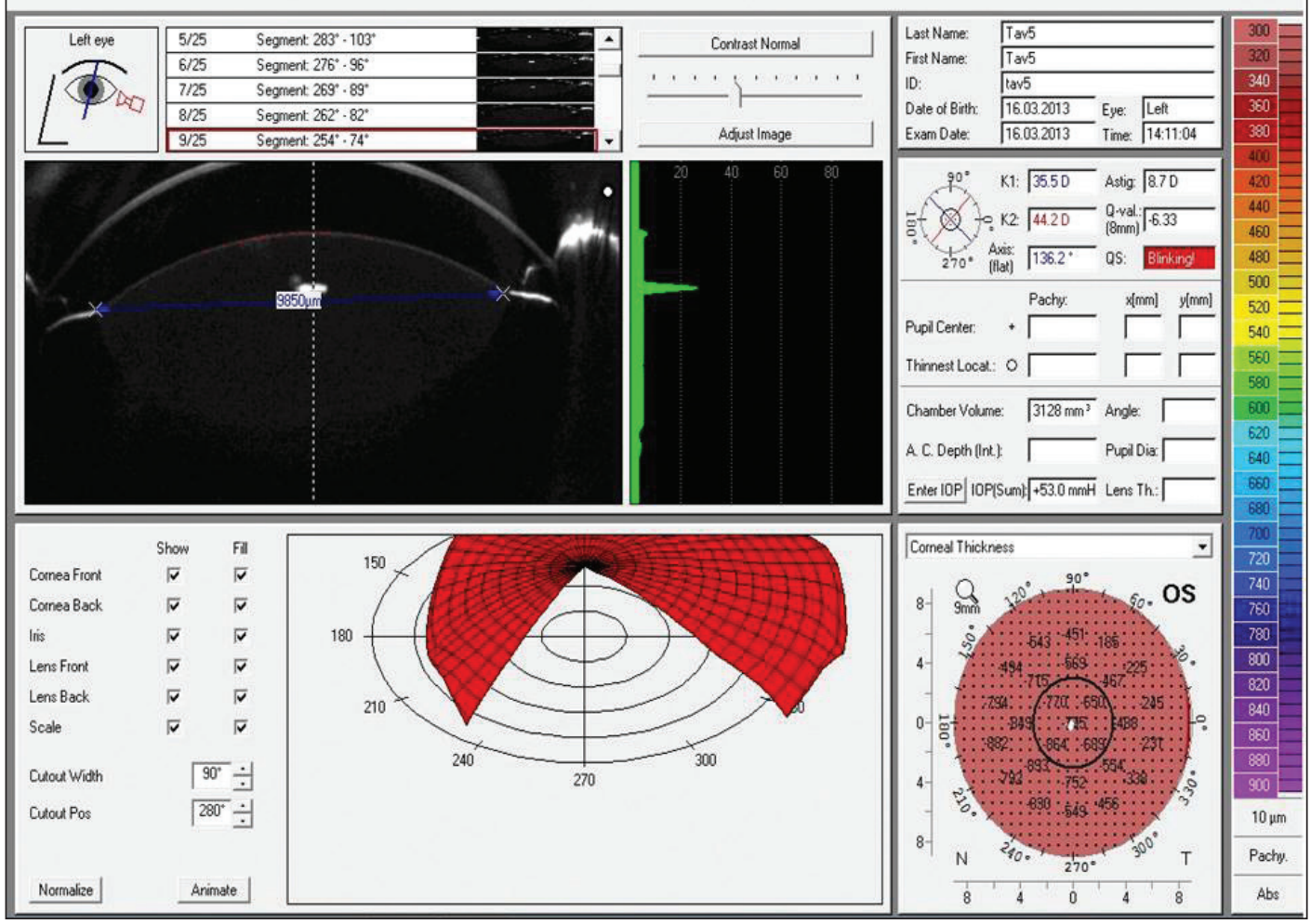

Figure 1: Pupil diameter values were measured as $9850 \pm 610 \mu \mathrm{m}$ with the ocular tomography device in all groups before the experiment.

\section{OCULUS - PENTACAM}
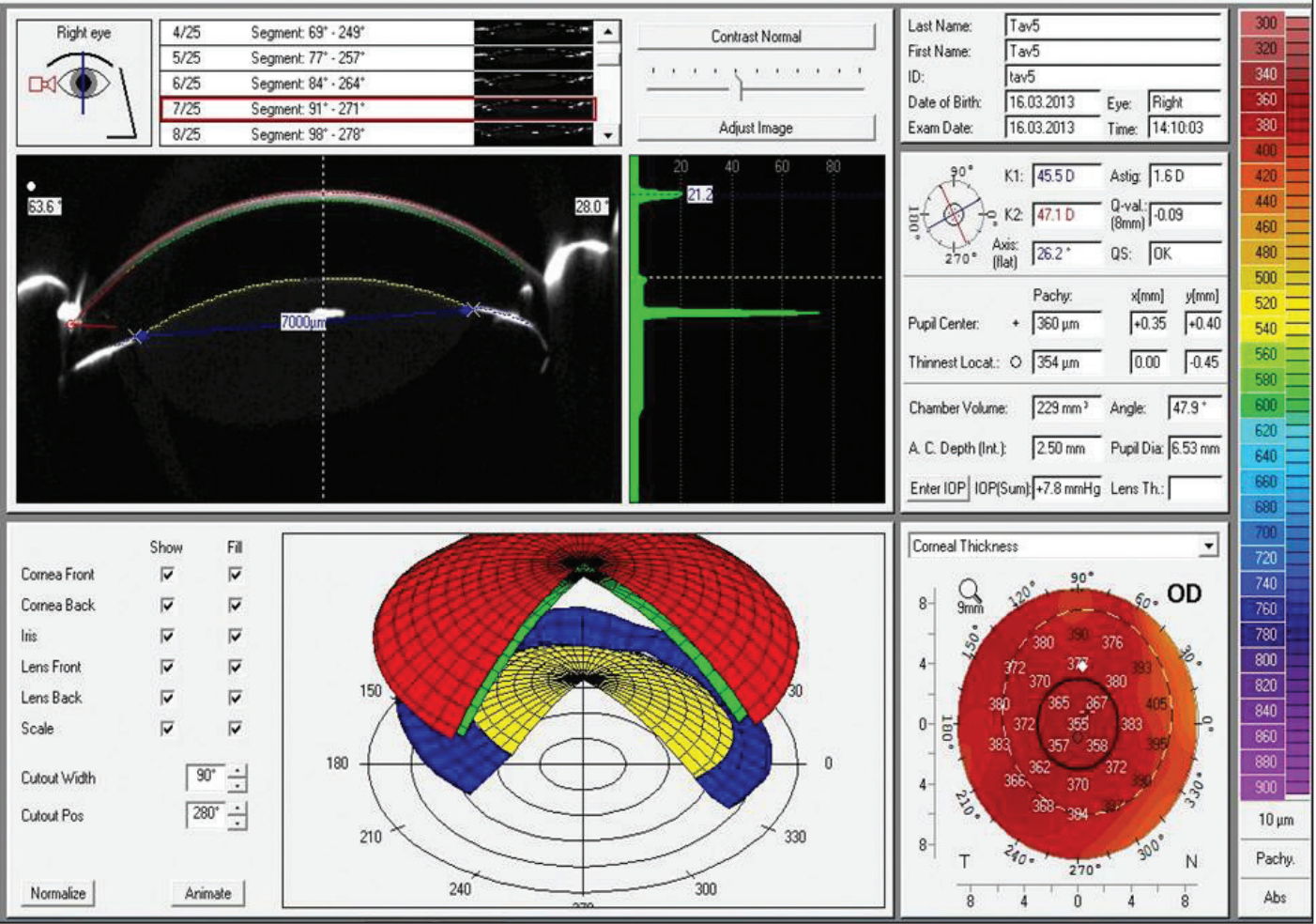

Figure 2: Pupil diameter values were measured as $7010 \pm 440 \mu \mathrm{m}$ with the ocular tomography device in the study group just after the experiment. 


\section{OCULUS - PENTACAM}

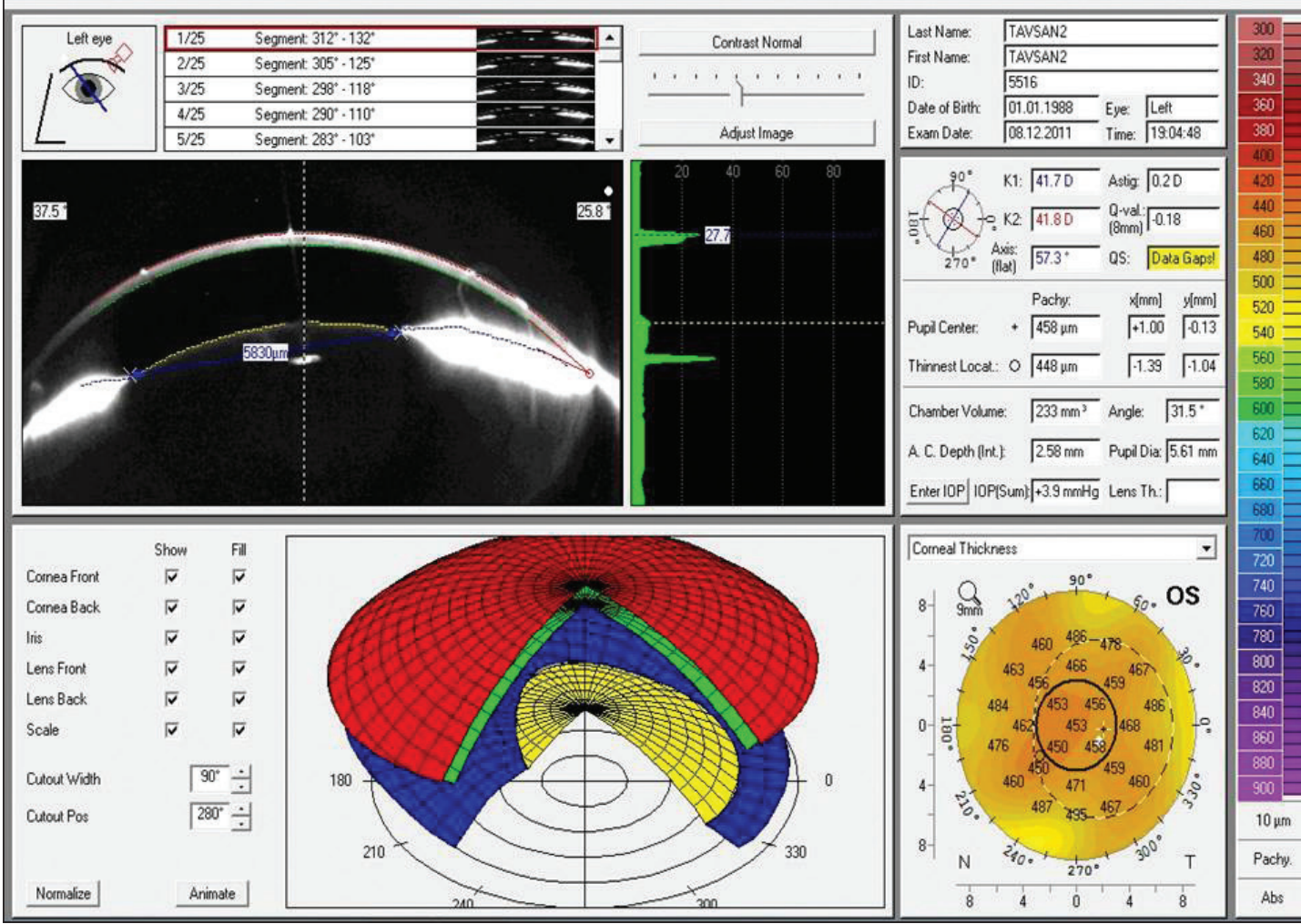

Figure 3: Pupil diameter values were measured as $5180 \pm$ $370 \mu \mathrm{m}$ with the ocular tomography device in all animals with $\mathrm{SAH}$.
There was an inverse relationship between the neuron density of the thoracic sympathetic center and pupil diameters $(p<0.005)$. Highly degenerated neuron density of the thoracal sympathetic center should also be considered an important factor in the development of a miotic pupil in both normal conditions and in various neurological pathologies that affect the light reflex (Figures 1,2).

\section{DISCUSSION}

The timely detection of neurological deterioration is important for patients with SAH. The most meaningful way to follow a traumatic or nontraumatic brain-injured patient in the intensive care unit is to perform serial bedside neurological examinations (26). In these examinations, pupil diameters should be checked daily in patients with SAH. Patients with central, preganglionic, or postganglionic lesions consistently show differences in pupil functions. The sympathetic system takes a role in the pupil-dilating phase of the edge-light pupil cycle time, presumably by exerting a tonic mydriatic effect. Since this tonic effect is lost in Horner's syndrome, leading to slower redilatation, the edge-light pupil cycle time becomes prolonged (5).

\section{Anatomo-Pathological Causes of Sympathetic Disorders of the Pupil:}

Horner's syndrome, which is seen when there is disruption of the oculosympathetic pathway, includes an injury influencing the ocular sympathetic nerve causing miosis, palpebral ptosis and enophthalmos, and is accompanied by hemifacial anhidrosis in its complete forms. Syringomyelia is a disorder of the spinal cord in which a cyst formation occurs within the cord. This is a significant reason of Horner's syndrome as a result of a lesion of the cervical sympathetic nerve fibers (13). Untreated contused cervicothoracic cords cause permanent sympathetic injury and result in Horner's syndrome, with the lesion localized to cranial to C5 cervical intumescence at the levels of C6-T2 $(7,15)$. Acute SAH surrounding the brain and spinal cord is an important cause of undiagnosed Horner's syndrome. SAH results in denervation injury-related neurodegeneration in the $\mathrm{CG}$ which induces indirect mydriasis (2). Highly degenerated neuron density of the CG and high neuron density of pupil-dilatory superior cervical sympathetic ganglia should be thought an essential factor for pupil dilatation (19). The loss of trophic effect from the presynaptic supply of the CG via the preganglionic fibers of the oculomotor nerve could potentially change the structure of the ganglion through the anterograde transneuronal degradation process in $\mathrm{SAH}$-induced ischemic degradation in the oculomotor nerve in the brainstem (4). Although Onen et al. reported that spinal cord ischemia could be responsible for sympathetic center ischemia-related miosis, the main neuropathological mechanism of ciliospinal center ischemia-based miosis is not yet known (19). Nevertheless, Kanat et al. showed that spinal SAH resulted in severe spinal cord ischemia causing neurodegeneration in the DRG via vasospasm of dorsal root ganglion-supplying arteries (12). Vasospastic blood vessels of the spinal cord come across to subarachnoid blood and 


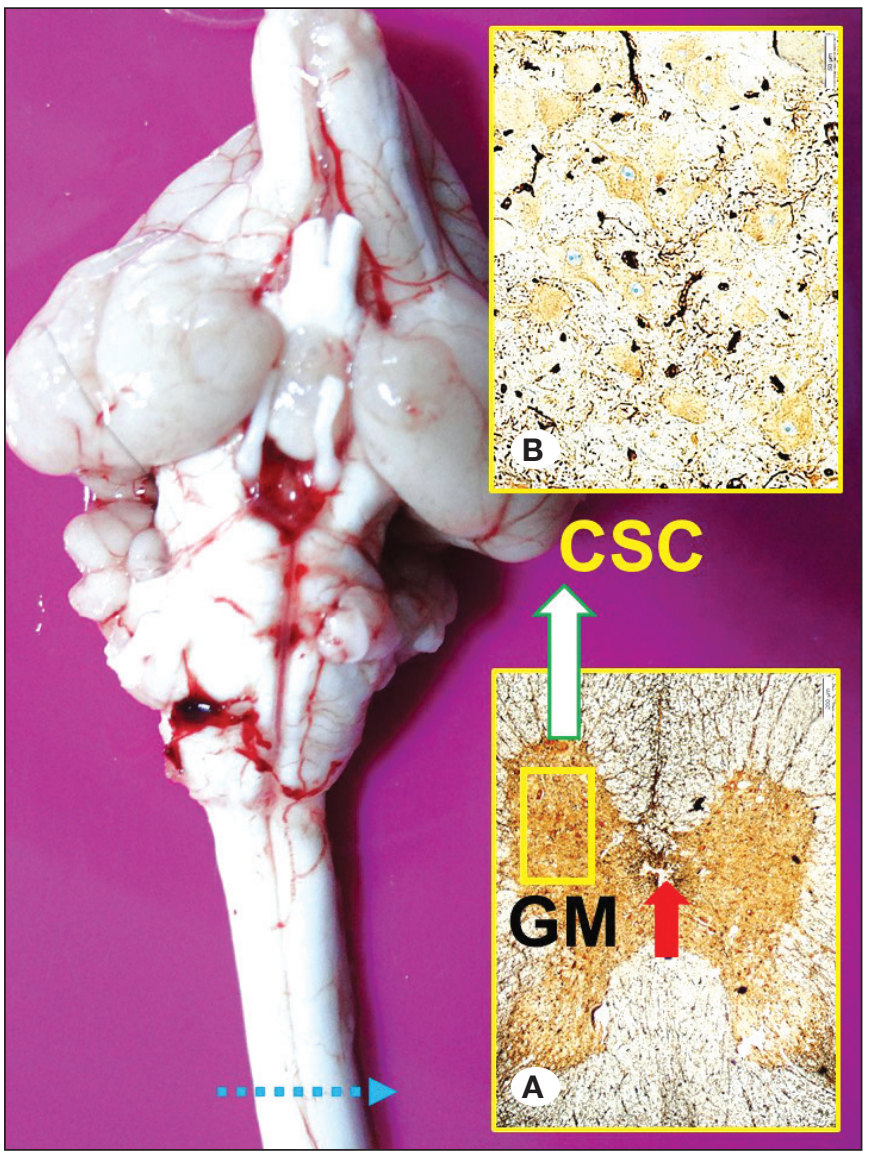

Figure 4: Macroscopical appearance of brain with cervicothoracic spinal cord to Th3 level (Base). Inlet; A: Horizontal section of spinal cord at Th1-level, central canal (red arrow), gray matter of spinal cord (GM), ciliospinal center (CSC-Yellow Square) (light microscopy, GFAP, x10). Inlet; B: Ciliospinal center neurons (light microscopy, GFAP, x 20) (B) are seen in a normal rabbit. vasospasm of DRG blood vessels may cause ischemic neurodegeneration in the DRG, while diminished normal neuron density or increased degraded neuron density of DR ganglia may play an essential role in the modulation of spinal cord blood flow. Low neuron density of DR ganglia should be considered an essential factor in the pathogenesis of severe neurodegeneration in ciliospinal center ischemia in SAH (12). Lee et al. reported that cervical spinal cord stimulation increases global cerebral and spinal blood flow (14). This information may prove that DR ganglia could have a vasodilatory effect through the electrical impulses on the spinal cord supplying arteries following SAH. Neuroprosthetic stimulations relieve volitional control of paretic eyes (16).

\section{Limitations:}

$\mathrm{SAH}$ results in bloody cerebrospinal fluid and the bloody or highly proteinous CSF may lead to neural degeneration (18). Spinal arachnoiditis may develop after SAH and intrathecal drug applications (18), and affects the spinal cord and nerve roots, and can also lead to spinal cord dysfunction (18). In this study, the saline injection by itself led to a slight to moderate diameter changes of the pupils. Those changes were prominent in animals of the SAH group. Saline injection in the sham group can be harmful, and lead to some changes in the subarachnoid space. In a study, saline injection directly into the sciatic nerve caused nerve damage (6).

\section{CONCLUSION}

The management of $\mathrm{SAH}$ requires a comprehensive understanding of the pathophysiology, which is paramount to define treatment strategies and algorithms (21). During the past 2 decades, neuroscientists have gained an improved understanding of the pathophysiological events that occur after SAH (9). This study demonstrates that SAH-induced ciliospinal center neurodegeneration may have been

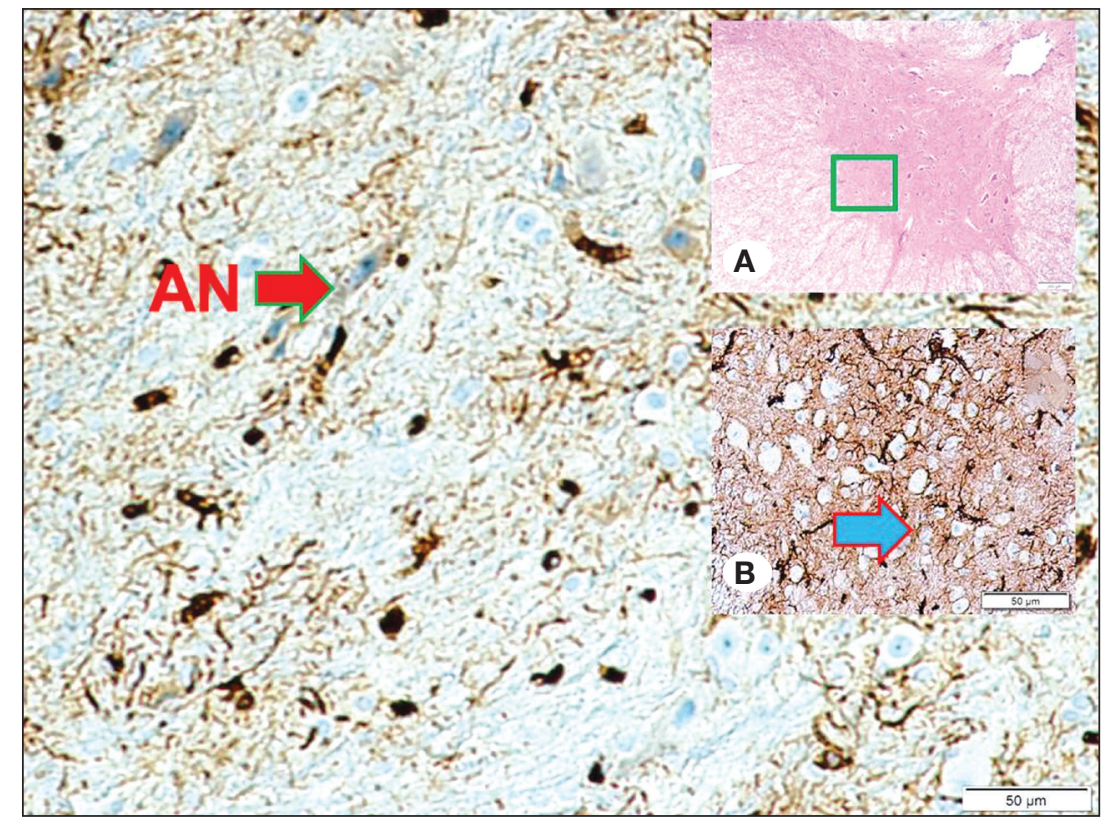

Figure 5: The apoptotic neurons (AN) of the ciliospinal center (Base) (light microscopy, TUNEL, $x$ 20). Inlet; A: histological appearances of the spinal cord at the level of Th-1 (light microscopy, Hematoxylin \& Eosin, x10). Inlet; B: the deformed neurons labeled with a blue arrow (light microscopy, GFAP, x10). 
responsible for permanent miosis following $\mathrm{SAH}$ and has not been mentioned in the literature so far. Further studies are needed.

\section{REFERENCES}

1. Aydin MD, Kanat A, Yilmaz A, Cakir M, Emet M, Cakir Z, Aslan S, Altas S, Gundogdu C: The role of ischemic neurodegeneration of the nodose ganglia on cardiac arrest after subarachnoid hemorrhage: An experimental study. Exp Neurol 230(1):90-95, 2011

2. Aydin MD, OnderS, Ulvi H, Onder A, Baykal O: Histopathological alterations in ciliary ganglions in meningitis: An experimental study. Minim. Invasive Neurosurg 48:297-301, 2005

3. Aydin MD, Akyol-Salman I, Sahin O: Histopathological changes in ciliary ganglion of rabbits with subarachnoid hemorrhage. Int J Neurosci 115:1595-1602, 2005

4. Aydin N, Kotan D, Keles S, Ondas O, Aydin MD, Baykal O, Gundogdu B: An experimental study of the neurophysical mechanisms of photophobia induced by subarachnoid hemorrhage. Neurosci Lett 630:93-100, 2016

5. Blumen SC, Feiler-Ofry V, Korczyn AD: The pupil cycle time in Horner's syndrome. J Clin Neuroophthalmol 6:232-235, 1986

6. Bostan H, Cabalar M, Altinay S, Kalkan Y, Tumkaya L, Kanat A, Balik S, Erkut A, Altuner D, Salihoglu Z, Kocer A: A histopathological examination: The sciatic nerve injury following analgesic drug injection in rats. North Clin Istanbul 5:176-185, 2018

7. Cagan E, Sayin R, Dogan M, Peker E, Cagan HH, Caksen $\mathrm{H}$ : Bilateral brachial plexus palsy and right Horner syndrome due to congenital cervicothoracal syringomyelia. Brain Dev 32:595-597, 2010

8. Gasenzer ER, Kanat A, Ozdemir V, Neugebauer E: Analyzing of dark past and bright present of neurosurgical history with a picture of musicians. Br J Neurosurg 32(3):303-304,2018

9. Kanat A: Brain oxygenation and energy metabolism: Part 1-Biological function and pathophysiology. Neurosurgery 52: 1508-1509; author reply 1509, 2003

10. Kanat A, Aydin MD: Asymptomatic familial cerebral aneurysms. Neurosurgery 44:1364-1365, 1999

11. Kanat A, Turkmenoglu O, Aydin MD, Yolas C, Aydin N, Gursan N, Tumkaya L, Demir R: Toward changing of the pathophysiologic basis of acute hydrocephalus after subarachnoid hemorrhage: A preliminary experimental study. World Neurosurg 80:390-395, 2013

12. Kanat A, Yilmaz A, Aydin MD, Musluman M, Altas S, Gursan $\mathrm{N}$ : Role of degenerated neuron density of dorsal root ganglion on anterior spinal artery vasospasm in subarachnoid hemorrhage: Experimental study. Acta Neurochir (Wien) 152: 2167-2172, 2010
13. Kerrison JB, Biousse V, Newman NJ: Isolated Horner's syndrome and syringomyelia. J Neurol Neurosurg Psychiatry 69:131-132, 2000

14. Lee JY, Huang DL, Keep R, Sagher O: Effect of electrical stimulation of the cervical spinal cord on blood flow following subarachnoid hemorrhage. J Neurosurg 109:1148-1154, 2008

15. Ma H, Kim I: Horner Syndrome associated with a Herniated Cervical Disc: A case report. Korean J Spine 9:108-110, 2012

16. Mondello SE, Kasten MR, Horner PJ, Moritz CT: Therapeutic intraspinal stimulation to generate activity and promote longterm recovery. Front Neurosci 8:21, 2014

17. Musluman AM, Aydin MD, Yilmaz A, Cansever T, Kanat $A$, Gundogdu C, Cakir Z, Emet M, Gursan N, Aydin N, Unal B: The effect of degenerated neuron density of petrosal ganglion on the development of blood pressure variabilities after subarachnoid hemorrhage in a rabbit model: An experimental study. Turk Neurosurg 21:559-566, 2011

18. Onder A, Serarslan Y, Aydin MD, Kanat A, Gursan N, Yolas C: Spinal arachnoiditis and the axonal degeneration of the spinal nerve roots due to ischemia induced by vasospasm: An experimental study. J Neurol Sci 26, 2009

19. Onen MR, Yilmaz I, Ramazanoglu L, Aydin MD, Keles S, Baykal O, Aydin N, Gundogdu C: Uncovering of the forgotten effect of superior cervical ganglia on pupil diameter determination in subarachnoid hemorrhage: Experimental study. Turk Neurosurg 28(1):48-55, 2016

20. Ozdemir NG, Aydin MD, Yolas C, Kanat A, Levent A, Gundogdu C, Aydin N: Predictive role of external carotid artery vasospasm on cerebral ischemia after subarachnoid hemorrhage: Experimental study. Turk Neurosurg 27:874-883, 2017

21. Ozturk C, Ozdemir NG, Kanat A, Aydin MD, Findik H, Aydin N, Kabalar ME, Kazdal H, Yolas C, Baykal O, Calik M: How reliable is pupillary evaluation following subarachnoid hemorrhage? Effect of oculomotor nerve degeneration secondary to posterior communicating artery vasospasm: First experimental study. J Neurol Surgery Part A Cent Eur Neurosurg 79: 302-308, 2018

22. Yilmaz A, Aydin MD, Kanat A, Musluman AM, Altas S, Aydin Y, Calik M, Gursan N: The effect of choroidal artery vasospasm on choroid plexus injury in subarachnoid hemorrhage: Experimental study. Turk Neurosurg 21:477-482, 2011

23. Yolas C, Kanat A, Aydin MD, Turkmenoglu ON, Gundogdu C: Important casual association of carotid body and glossopharyngeal nerve and lung following experimental subarachnoid hemorrhage in rabbits. First report. J Neurol Sci 336: 220-226, 2014 